\title{
La economía de China en el contexto de la crisis económica mundial 2007-2009¹
}

DOI: $10.32870 /$ mycp.v13i39.348

Roberto Hernández Hernández ${ }^{2}$

\section{Resumen}

$\mathrm{E}$ 1 impacto de la crisis económica mundial de 2007-2009 sobre la economía de China ha sido breve y limitado. Si lo comparamos con lo acontecido en la mayoría de las economías del resto del mundo, China ha destacado por la rapidez con que "tocó fondo" y restableció las tasas de crecimiento económico similares a la etapa anterior a la crisis.

Ante este conflicto de carácter económico global, el gobierno de China mostró una gran capacidad de reacción. A través de la aplicación de una política netamente keynesiana puso en marcha una gran cantidad de recursos financieros para apoyar la obra pública; frente a la reducción de las exportaciones, impulsó la demanda interna sustentada en el gasto fiscal.

China ha salido fortificada del proceso. Su relación productiva con las economías del este de Asia le ha conferido un estatus de liderazgo en el manejo de los asuntos económicos y estratégico-políticos de la región. Sin embargo, ello no implica que sus problemas de desigualdad social y de bajo poder adquisitivo de una gran parte de la población hayan sido solucionados. Prácticamente no existe paralelo con ningún país que sea tan grande y tan dominante en términos absolutos, y que al mismo tiempo esté tan rezagado en términos de ingreso per cápita y otros indicadores de desarrollo.

Palabras clave: China, crisis económica, Economía Política.

1. El autor agradece el apoyo de la estudiante de la licenciatura en Estudios Internacionales Lorena Angélica Velázquez Guerrero en la recopilación y procesamiento de datos estadísticos.

2. Profesor-Investigador del Departamento de Estudios del Pacífico, Centro Universitario de Ciencias Sociales y Humanidades, Universidad de Guadalajara; miembro del SNI. ORCID http://orcid.org/0000-0002-9145-5865 


\section{Abstract}

The impact of the global economic crisis of 2007-2009 on the Chinese economy has been brief and limited. When compared to what happened in most economies around the world, China has been remarkable for the speed with which "bottomed out" and restored economic growth rates similar to pre-crisis stage.

Given this global economic conflict the Chinese government showed great resilience. Through the implementation of a purely Keynesian policy launched a huge amount of financial resources to support public works. Since the decline of exports, it boosted domestic demand.

China has emerged fortified from the process. Their productive relationship with the economies of East Asia has given it a leadership status in the management of economic affairs and strategic-political in the region. However, this does not mean that their problems of social inequality and low purchasing power of a large proportion of the population have been solved. There is virtually no parallel of any country which is so big and so dominant in absolute terms, and at the same time is so far behind in terms of income per capita and other indicators of development.

Keywords: China, economic crisis, Political Economy.

\section{Introducción}

El impacto de la crisis económica mundial de 2007-2009 sobre la economía de China ha sido breve y limitado; sobre todo si lo comparamos con lo acontecido en la mayoría de las economías del resto del mundo. En este sentido, China ha sido el país que mejor ha salido librado de esta crisis económica. Los medios especializados en asuntos de Asia, desde principios de 2010, al referirse a la rapidez y la forma en que China salió de la crisis, la refieren como un "rebote" y así lo confirman los principales indicadores económicos, tales como el del crecimiento del producto interno bruto (PIB), el comercio exterior y la inversión extranjera directa.

Por su parte, la economía de los Estados Unidos, donde se originó la crisis, ha sido de las que más han sufrido las consecuencias de la depresión económica, en especial por sus efectos en los sectores financiero y de vivienda, que se extendieron hacia el resto de la economía. Europa occidental y las economías 
más avanzadas de Asia, también se han visto perjudicadas por el colapso del comercio mundial y el agravamiento de sus propios problemas financieros. Esta circunstancia incidió negativamente en algunos indicadores básicos de la economía de China, en especial sobre su comercio exterior.

De acuerdo con el Departamento Nacional de Investigación Económica de los Estados Unidos, la crisis económica inició en diciembre de 2007 y se prolongó durante 18 meses hasta junio de 2009. Señaló que ésta ha sido la re-

China ha destacado por la rapidez con que "tocó fondo" y restableció las tasas de crecimiento económico similares a la etapa anterior a la crisis cesión más larga en los Estados Unidos desde la Gran Depresión y advirtió, además, que cualquier declinación económica posterior podría ser parte de una nueva recesión y no una continuación de la iniciada en 2007 (LA Times, 2010; NY Times, 2010b).

A lo largo de este difícil periodo para la economía mundial, China ha destacado por la rapidez con que "tocó fondo" y restableció las tasas de crecimiento económico similares a la etapa anterior a la crisis. El crecimiento del producto interno bruto (PIB) que en 2007 había sido de 13.0\%, disminuyó a 8.1\% en 2009. Sin embargo, para 2010 las expectativas son que alcance un PIB de entre $9.6 \%$ y $10 \%$ por ciento anual. China ha sorteado la crisis financiera y económica global mejor que muchas otras economías, gracias a que no depende del financiamiento externo, por lo que sus bancos han salido prácticamente indemnes de la turbulencia financiera internacional, y a que cuenta con un suficiente margen de maniobra fiscal y macroeconómica para aplicar vigorosas medidas de estímulo a la economía (World Bank, 2010).

Este trabajo presenta un repaso general de la crisis financiera y económica mundial, la forma y la intensidad en que impactó a China y las políticas aplicadas por el régimen para salir adelante. El trasfondo de la Economía Política para explicar este tema, queda de manifiesto a lo largo del artículo; sobre todo, en cuanto a la estrecha relación existente entre el mercado y el Estado, en los términos planteados por Robert Gilpin (1996: 269-285). 


\section{La crisis económica mundial de 2007-2009}

Por crisis económica global del 2007-2009 se entiende al conjunto de problemas económicos que tuvieron su origen en los Estados Unidos y que afectaron, inicialmente y de manera muy intensa, al sector financiero en general e hipotecario en particular de ese país, para después extenderse a la economía real y provocar una recesión a nivel mundial. Esta crisis se originó por la falta de confianza en el sistema financiero, dada la sobrevalorización especulativa de algunos bienes (en especial los bienes raíces), así como en los altos precios de las materias primas (Baily y Elliot, 2009). La dimensión de la crisis económica mundial y la forma como impactó a algunas regiones y países más importantes de la economía mundial, se aprecian en el cuadro 1. Como podemos notar, las principales economías del mundo, con excepción de la de China e India, tuvieron una significativa disminución en su producto interno bruto. También podemos ver cómo a partir de 2010 se ha dado una ligera recuperación de la economía mundial.

De acuerdo con un informe del Banco Mundial (2010), desde principios de este año, la fase aguda de la crisis financiera ha pasado y está en marcha una recuperación económica mundial. Sin embargo - como el mismo informe advierte-, la recuperación es frágil y se espera que disminuya su ritmo en la segunda mitad de 2010 a medida que se desvanezca el impacto de las medidas fiscales y monetarias y el presente ciclo de inventarios siga su curso. De hecho, el ritmo de crecimiento de la producción industrial ya está menguando (si bien desde tasas muy altas). En consecuencia, el crecimiento del empleo también se mantendrá débil, y se prevé que el desempleo presente tasas relativamente altas por muchos años. La fortaleza total de la recuperación y su durabilidad dependerán del grado en que la demanda de las familias y el sector empresarial se fortalezca en el corto plazo. Las cifras sobre el crecimiento mundial en 2010 rondan en alrededor de $2.7 \%$ y 3.2\% para 2011, después de un descenso a $2.2 \%$ en 2009. Sin embargo, no se descarta un escenario doble, en el que se mantuviera un bajo crecimiento en 2011 o un fortalecimiento en la recuperación.

El Fondo Monetario Internacional (IMF, 2010), por su parte, proyecta tasas de crecimiento económico mundial de $4.5 \%$ para 2010 y de $4.25 \%$ para 2011; aunque también advierte que se mantienen latentes los riesgos de una disminución en la actividad económica, ante el resurgimiento de las turbulencias financieras y señala que los resultados dependerán de la aplicación de 


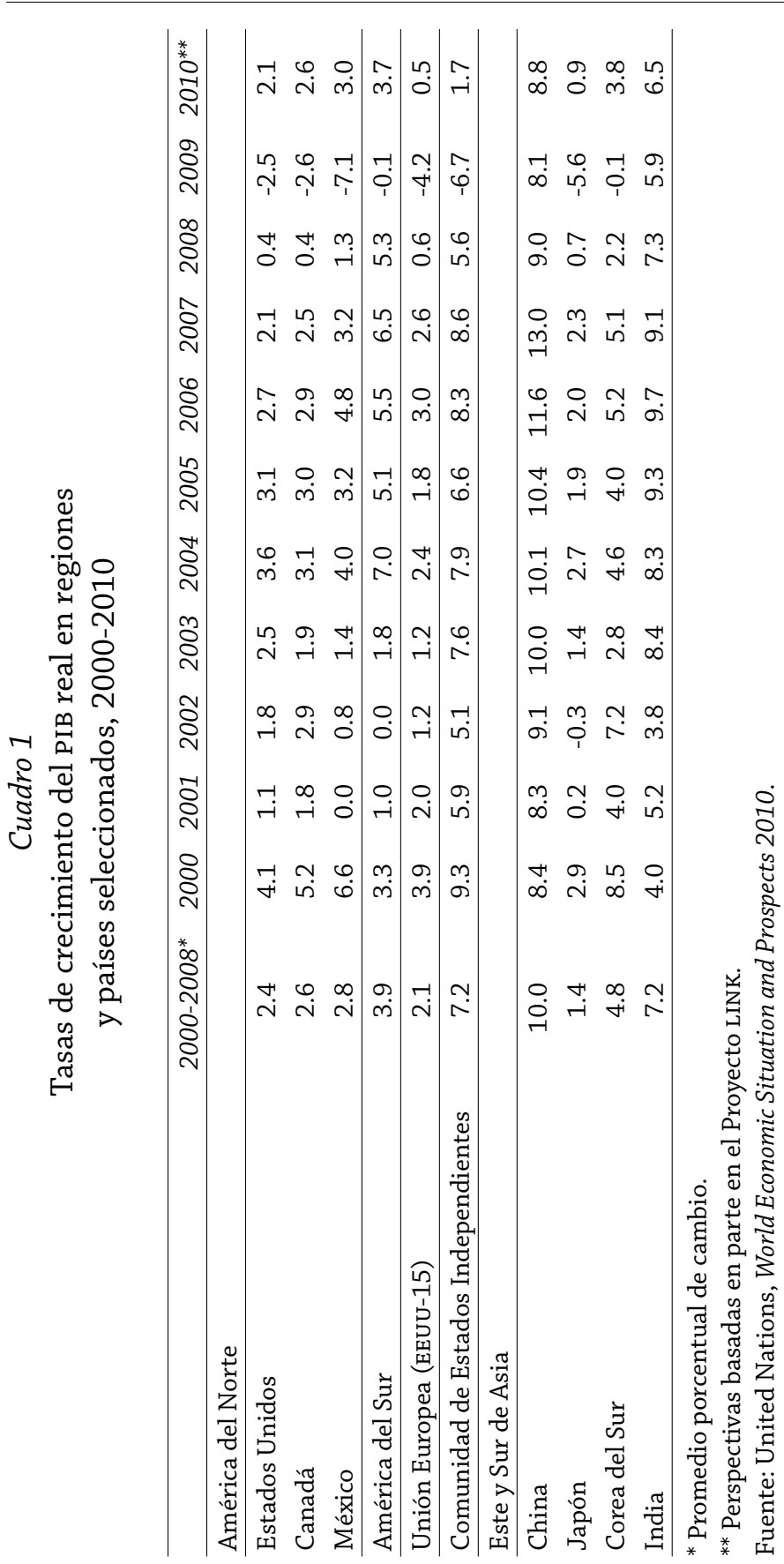


políticas orientadas a restablecer la confianza y la estabilidad, particularmente en la zona del euro.

Los mercados financieros se han estabilizado y se recuperan, pero permanecen débiles. La liquidez interbancaria, medida por la diferencia entre las tasas de interés que los bancos comerciales se aplican entre sí y lo que tienen que pagar a los bancos centrales, ha descendido de un pico sin precedentes de 366 puntos base en los mercados del dólar a menos de 15 puntos base, nivel cercano a su rango "normal", anterior a la crisis. Las monedas, que en todo el mundo se depreciaron ante el dólar en los días posteriores a la crisis, han recuperado en buena medida sus niveles anteriores. Y los flujos internacionales de capital hacia los países en desarrollo se han recuperado, con un rápido incremento durante los últimos meses de 2009. Asimismo, los costos de los préstamos para los deudores de mercados emergentes se han estabilizado en los trimestres pasados, pero permanecen elevados (Baily y Elliot, 2009).

La economía real también se recupera. La producción industrial, tanto en los países de ingreso alto como en las economías en desarrollo se expandió a una tasa anualizada superior a $12 \%$ en el tercer trimestre de 2009. A la fuerte caída en los inventarios durante la crisis, la estabilización de los niveles de éstos, ha contribuido a un fuerte repunte en la producción y se espera que este factor apoye la producción industrial, aun cuando las tasas de crecimiento económico no sean tan halagüeñas.

El comercio también se ha recuperado, aunque todavía permanece deprimido. Las tasas de crecimiento trimestral han presentado datos positivos en meses recientes, pero el valor del comercio en dólares estadounidenses es aún $17 \%$ menor que el nivel de septiembre de 2009. Los precios más bajos de los productos básicos demuestran que el volumen del comercio ha mejorado, pero aun así es de $3 \%$ inferior al de hace un año.

De acuerdo con el FMI (IMF, 2010), las medidas de política económica aplicadas por las economías avanzadas deberían centrarse en una consolidación fiscal que inspire confianza, en especial en políticas encaminadas a reforzar las perspectivas de crecimiento a mediano plazo, tales como las reformas al sector financiero y a los sistemas tributarios y de prestaciones, complementadas con reformas estructurales para fortalecer el crecimiento y la competitividad. En las economías emergentes las políticas también deberían ayudar a reequilibrar la demanda mundial, entre otras formas, mediante la aplicación de reformas estructurales y, en algunos casos, con una mayor flexibilidad del tipo de cambio. 


\section{La economía de China en contexto de la crisis mundial}

En los últimos 30 años el crecimiento económico de China, sustentado en la apertura económica, ha sido impresionante. De 1981 a 2009, el producto interno bruto (PIB) fue de 9.9\%; llegando a alcanzar en algunos años incrementos superiores a 15\% (Chinability, 2010). No obstante el crecimiento económico cercano a $10 \%$ anual, los altibajos han sido muy marcados, especialmente en 1989 y 1990, cuando el PIB disminuyó a tasas de $4.1 \%$ y 3.8\% anual, respectivamente. Otro periodo de tasas relativamente bajas fueron las 1997 a 1999 cuando reportaron niveles del PIB ligeramente inferiores a 8\% anual. Entre los años 2000 y 2008 las tasas de crecimiento económico volvieron a repuntar hasta alcanzar un promedio cercano a $10 \%$ anual durante este periodo (gráfica 1).

Cabe resaltar que, contrariamente a las expectativas y a las experiencias reportadas por otros países del este de Asia y sobre todo del resto del mundo (con algunas pocas excepciones), la disminución de la actividad económica en China fue poco significativa. De acuerdo con el Banco Mundial el PIB de China disminuyó a $8.7 \%$ en 2009, considerado el peor año de la crisis económica mundial. Para este año de 2010 se espera un nuevo aumento de 8 décimas de punto para alcanzar un PIB de $9.5 \%$ (cuadro 2 ). ${ }^{3}$

De hecho existe un gran optimismo por parte de los analistas a nivel mundial sobre el mantenimiento de la tendencia hacia un fuerte crecimiento económico por parte de China; aunque también se dan algunas inquietudes sobre las bases de la recuperación y la duración que pueda tener ésta. Para 2010 el Fondo Monetario Internacional prevé una tasa de crecimiento en el PIB de $10 \%$ (tal como se percibe en la gráfica 1) y Goldman Sachs la proyecta hasta $11.9 \%{ }^{4}$

3. Cabe señalar que, de nuevo, la realidad económica está superando las expectativas. Para septiembre de 2010 la Oficina Nacional de Estadísticas de China había reportado para la primera mitad del año, un aumento anualizado en el PIB de $11.1 \%$ (http://www.chinadalily.com.cn/ china/2010/7/15content_10110093.htm). La razón de este aumento radica en el fuerte consumo interno y la inversión apoyada en los estímulos gubernamentales.

4. Algunos de los datos sobre esta coyuntura económica pueden encontrarse en: The Economist, 15 de agosto de 2009: 70; Chang, G. Gordon, “China's 8.9\% Growt? No Way", Forbes, forbes. com. (20/11/2009); "IMF Revises Up Global Growth Forecasts", Roubini Global Economics $(22 / 04 / 2010)$. 
Gráfica 1

China, PIB 1981-2010

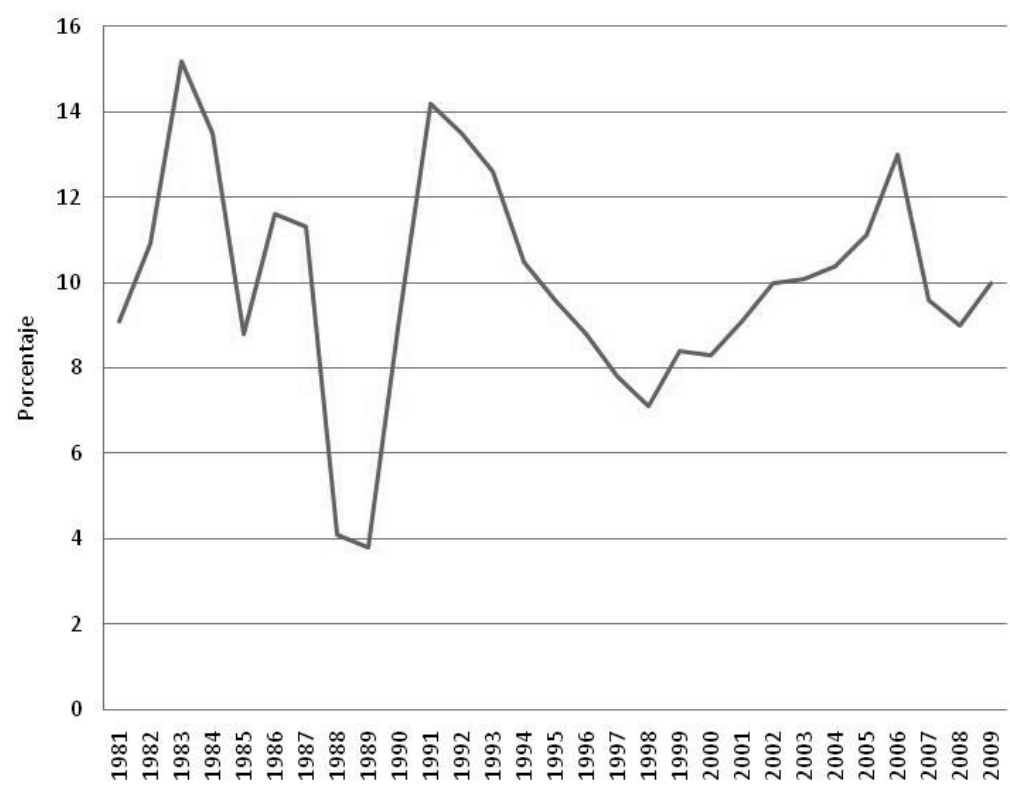

Fuente: National Bureau of Statistics, en: http://www.chinability.com/GDP.htm), fecha de consulta: 20/04/2010.<R> Para datos de 2000, 2005, 2007 y 2009, The World Bank (2010) Data profile, en: http://ddp-ext.worldbank.org/ext/ddpreports/ ViewSharedReport?\&CF=\&REPORT_ID=9147\&REQUEST_TYPE=VIEWADVANCED, fecha de consulta: 20/05/2010.

\section{Cuadro 2}

Producto interno bruto de China y el este de Asia, 1995-2012

\begin{tabular}{lccccccc}
\hline & $1995-2005$ & 2007 & 2008 & 2009 & 2010 & 2011 & 2012 \\
\hline China & 9.1 & 13 & 9.6 & 8.7 & 9.5 & 8.5 & 8.2 \\
Este de Asia, excluyendo a China & 3.5 & 6.2 & 4.7 & 1.5 & 5.8 & 5.3 & 5.6 \\
\hline
\end{tabular}

World Bank, Global Economic Prospects Summer 2010: Regional Annex, Washington, DC, 2010.

Este rápido crecimiento ha hecho que China pase de un PIB de 175,573.9 millones de dólares en 1979 (NationMaster, 2010) a 8.7 billones de dólares 
en 2009, en términos de paridad del poder adquisitivo (PPP) ${ }^{5}$, tal como se muestra en la gráfica 2.

En términos de dólares corrientes, el PIB de China fue en ese mismo año de 4.8 billones, ligeramente inferior al de Japón que reportó 5.1 billones (véase grafica 3). ${ }^{6}$ Sin embargo, a mediados de agosto de 2010 las principales agencias informativas dieron a conocer que, en parte, gracias a la crisis económica mundial, la economía de China se ha convertido en la segunda a nivel mundial. ${ }^{7}$

Al impresionante crecimiento económico de China de los últimos años hay que agregar que Japón, junto con la mayoría de las economías en el mundo, se ha visto seriamente afectado en sus expectativas de crecimiento económico debido a la crisis.

No obstante que China se ha convertido en una potencia económica, en términos de poder adquisitivo per cápita continúa muy por debajo de muchos países, tal como se puede apreciar en las gráficas 4 y 5 y en los datos del FMI. ${ }^{8}$ Dicho de otra forma, China requiere cuatro veces más de mano de obra que

5. Sobre la base del PPP, que asume costos similares para productos y servicios iguales en diferentes países, el PIB de China fue superior al de Japón en 2001 y sobrepasará al de EEUU en 2020.

6. Ello significa que la economía de China ha aumentado en más de 10 veces al nivel que tenía hace de 30 años.

7. La crisis económica mundial contribuyó a este hecho, dado que mientras Japón en 2008 y 2009 tuvo una disminución en el crecimiento económico de 0.7 y 5.6 respectivamente, China por el contrario reportó un crecimiento de 9.0 y 8.1 respectivamente (UN, 2010). Específicamente, en el segundo trimestre de 2010 el producto interno bruto de Japón totalizó 1.288 billones de dólares, menos que los 1.337 billones de dólares de China. Aunque si se toma en cuenta todo el primer semestre del año, el PIB de Japón continúa siendo superior con 5.07 billones de dólares, frente a los 4.9 billones alcanzados por China. De seguir la tendencia actual China superaría a EEUU en términos de PIB, medido en dólares corrientes, para 2027. Esto de acuerdo con Jim O’Neill, economista en jefe de Goldman Sachs Group Inc. Véase: Bloomberg News, "China GDP Surpasses Japan, Capping Three-Decade Rise” (http://www.businessweek. com/news/2010-08-16/china-gdp-surpasses-japan-capping-three-decade-rise.html, fecha de consulta: 1/09/2010); Blair, C. Dennis, "Annual Threat Assessment of the us Intelligence Community for the Senate Select Committee on Intelligence", Office of the Director of $\mathrm{Na}$ tional Intelligence, EEUU, 2010.

8. Según con datos del FMI el PIB per cápita de China para el año 1990 fue de 796.108 dólares estadounidenses, durante casi todos los años noventa se mantuvo por debajo de los dos mil dólares; en 1998 alcanzó 1,997 dólares. Hasta 1999 se superaron los dos mil dólares con una cifra de 2,163 dólares estadounidenses. Fuente: World Economic Outlook Database (2010), International Monetary Fund. (http://www.imf.org/external/pubs/ft/weo/2010/01/weodata/ weorept.aspx? sy $=1990 \&$ ey $=1999 \& \mathrm{scsm}=1 \& \mathrm{ssd}=1 \&$ sort $=$ country\&ds $=. \& \mathrm{br}=1 \& \mathrm{c}=924 \& \mathrm{~s}=\mathrm{PP}$ PPC\&grp $=0 \& a=\& p r 1 . x=59 \& p r 1 . y=5(31 / o 5 / 2010)$ 
Gráfica 2

Producto interno bruto en PPP* de las economías del este de Asia y México, 2009

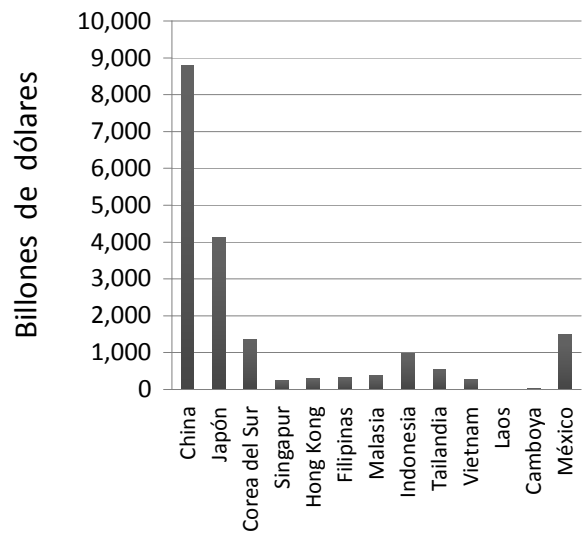

*Purchasing power parity

Fuente: Elaboración propia con base en

The World Factbook, varios países, 2010, cIA, en: http:// www.cia.gov, fecha de consulta: 23/04/2010.
Gráfica 3

Producto interno bruto de las economías del este de Asia y México 2009

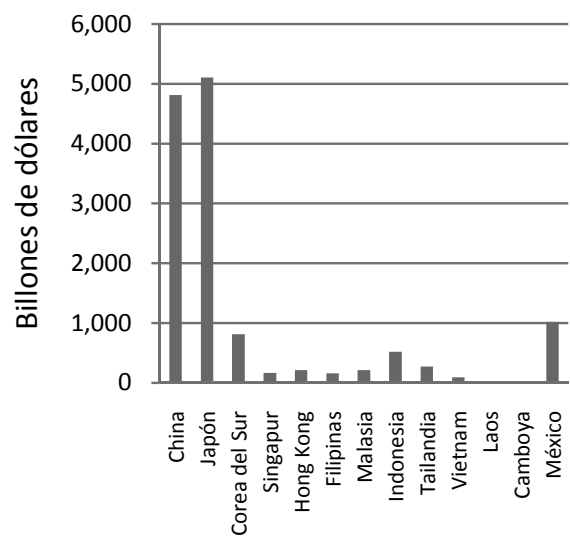

Tasa de cambio oficial.

Fuente: Elaboración propia con base en, The World Factbook, varios países, 2010, cIA, en: http:// www.cia.gov, fecha de consulta: 23/04/2010.

los EEUU para producir menos de la mitad del producto de los EeUU, lo cual suena un tanto engañoso, dado que la productividad en China no está muy por debajo de la de los EEUU. Sin embargo, la reducida aportación al PIB por parte del cientos de millones de pobres de las zonas rurales, hacen que las cifras de producción per cápita sean muy bajas (The Economist, 2010). 
Gráfica 4

Producto interno bruto en PPP*

per cápita de las economías

del este de Asia y México 2009

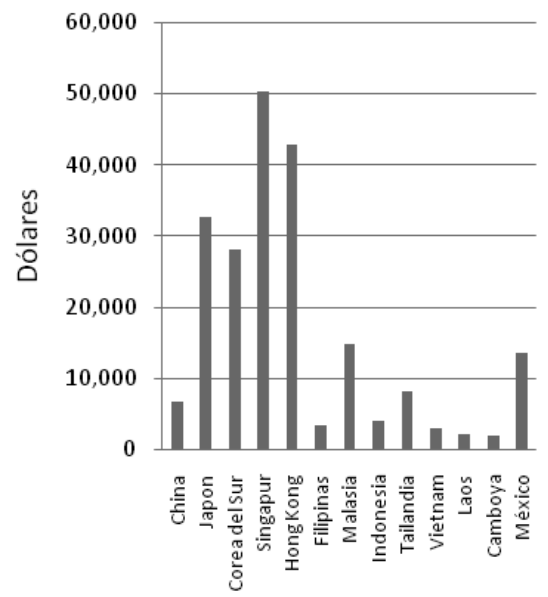

Fuente: *Purchasing Power Parity.

Fuente: elaboración propia con base en The

World factbook, varios países, 2010, cIA, en: http://www.cia.gov. Fecha de consulta: 23/04/2010.

\section{Gráfica 5}

Producto interno bruto per cápita de las economías del este de Asia y México

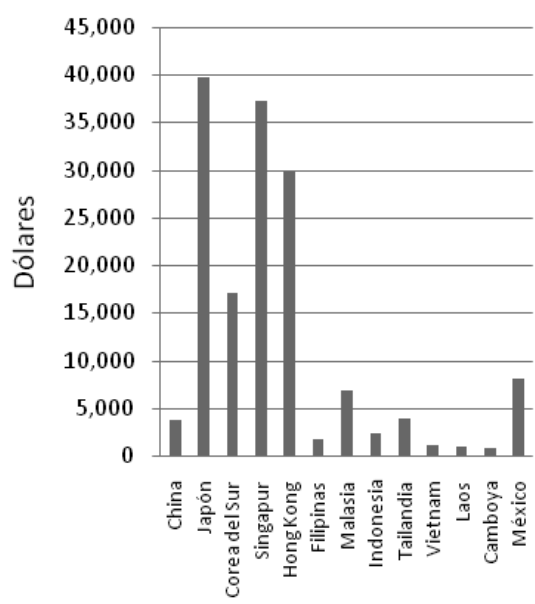

* Tasa de cambio oficial.

Fuente: elaboración propia con base en World Economic Outlook database, abril 2010, IMF, en: http://imf.org. Fecha de consulta: 30/04/2010.

Tal como afirma Eswar Prasad de la Universidad de Cornell: virtualmente no existe paralelo en la historia de ningún país que sea tan grande y tan dominante en términos absolutos, y que al mismo tiempo esté tan rezagado en términos de ingreso per cápita y otros indicadores de desarrollo. Por ello, aun manteniendo los niveles de crecimiento económico actual, a China le tomará una generación para alcanzar el nivel de desarrollo de las economías avanzadas (The Economist, 2010).

\section{Comercio exterior}

En términos de comercio exterior, la economía de China, al igual que las del resto del mundo, se vio afectada con la crisis económica de 2007-2009. Esto se debió a la dependencia que China tiene de las exportaciones. Por ello, ante 
la caída de la demanda global -en especial de los mercados de Estados Unidos y Europa- en 2009 se redujo drásticamente su comercio exterior (Yu, 2010). De acuerdo con cifras oficiales, el comercio exterior de China había pasado de aproximadamente 20,000 millones de dólares a finales de los años setenta a 1.76 billones en 2006. En 2008 alcanzó un pico de 1.43 billones de exportaciones y de 1.13 billones de importaciones, con un extraordinario superávit comercial de 256.3 mil millones de dólares. Sin embargo, en 2009 se presentó un fuerte descenso de $13.9 \%$ en la balanza comercial de China, siendo las exportaciones las más afectadas, con una reducción de 16\%; las importaciones, por su parte, disminuyeron en $11.2 \%$. Esta disminución representa un retroceso momentáneo en la tendencia de incremento anual de $20.8 \%$ logrado entre 2000 y 2009 (cuadro 3). Cabe señalar que no obstante esta disminución en 2009, el superávit se mantuvo y la balanza comercial en su conjunto no llegó a retroceder por debajo de los niveles de los años anteriores a 2008. Si bien la baja en el volumen del comercio exterior se relaciona con la caída de la demanda global, la contracción del comercio exterior de China no ha detenido su crecimiento relativo, si se compara con otros países netamente exportadores, por lo que en este contexto de crisis económica global, China se ha colocado como el mayor exportador del mundo (OMC, 2009). ${ }^{9}$

En lo que va de 2010, las exportaciones chinas están mostrando señales de una franca recuperación. En el primer cuatrimestre del año las exportaciones muestran un aumento de $28.7 \%$ (Xinhua, 2010) y de enero a agosto el superávit comercial alcanzó los 104.5 mil millones de dólares; aunque inferior a los años anteriores, no deja de ser significativo, puesto que muestra una recuperación de las exportaciones de China.

La alta dependencia de las exportaciones por parte de China es el resultado de su política de promoción de las exportaciones. El crecimiento de las exportaciones continúa siendo un importante componente que apoya el rápido crecimiento económico de China. Las exportaciones de bienes y servicios constituyen 39.7\% del PIB (China Balance of Trade, 2010). Las principales exportaciones de China son: máquinas de oficina y equipo para procesamiento de datos, equipo de telecomunicaciones, maquinaria eléctrica y ropa y prendas de

9. De acuerdo con datos de la OMC en 2009 el valor total de las exportaciones de Alemania fue de 1.12 billones de dólares y de 1.2 billones de dólares para China, con lo cual China desplazó a Alemania en este año como primer exportador mundial. Fuente: Organización Mundial de Comercio (2010), Estadísticas del Comercio Internacional: http://www.wto.org/spanish/ news_s/pres10_s/pr598_s.htm, fecha de consulta: 31/05/2010. 
vestir. Por otra parte, China importa principalmente materias primas: hierro y acero, petróleo y minerales combustibles; maquinaria y equipo, plásticos, equipo óptico y médico, así como químicos orgánicos. Sus principales socios comerciales son: la Unión Europea, los Estados Unidos, Japón, Hong Kong y Corea del Sur (Balance of Trade, 2010).

A este respecto, hay que tener en cuenta que, desde una perspectiva macroeconómica, la alta dependencia de China de las exportaciones es parcialmente atribuible a la sobrecapacidad, producto de la sobreinversión. En 2007, la contribución combinada de inversión en activos fijos (IAF) y las exportaciones netas al crecimiento del PIB fue superior a $60 \%$. Se considera que la IAF y las exportaciones son el cuarto de máquinas que impulsa el crecimiento económico de China. ${ }^{10}$

Un elemento prioritario en la estrategia del desarrollo económico de China -al igual que en muchas otras economías del este de Asia- es la orientación hacia las exportaciones. Los funcionarios chinos reconocen que la mitad del comercio exterior de China es conducido por empresas de inversión extranjera establecidas en China y que la mitad del comercio es de bienes procesados. Estos hechos hacen que la economía de China (y la de los demás países del este de Asia) dependan en gran medida de las exportaciones, en especial hacia el mercado de los Estados Unidos. ${ }^{11}$

\section{Inversión extranjera directa}

En cuanto a inversión extranjera directa (IED), China se ha convertido en uno delos principales destinos mundiales. Deun promedio anual de 1,800 millones de dólares captados durante el periodo de 1979 a 1989, la IED captada por China aumentó a un promedio de 68,500 millones de dólares anuales entre 1997 y

10. Yu (2010) analiza en detalle la relación entre la inversión en activos fijos (IAF) y el crecimiento del PIB. En el caso de China, el crecimiento de la IAF ha sido continuamente mayor que el crecimiento del PIB. Este nivel alto en la IAF impulsó el aumento del PIB, pero el exceso en la demanda (sobrecalentamiento) provocó una sobrecapacidad productiva que tenía que ser direccionada al exterior debido a que la demanda agregada interna no crecía al mismo ritmo de la IAF. Para prevenir el colapso del crecimiento económico, ante la caída de la demanda externa, había que atacar en dos frentes: reducir la IAF y/o aumentar la demanda agregada interna. La igualdad de la tasa de crecimiento con la de otros componentes de la demanda agregada es una condición necesaria y suficiente para el mantenimiento estable y sustentable en el largo plazo.

11. Sutter, Robert, "The Obama Administration and US Policy in Asia", Contemporary Southeast Asia, vol. 31, 2009, núm. 2, pp. 189-216. 


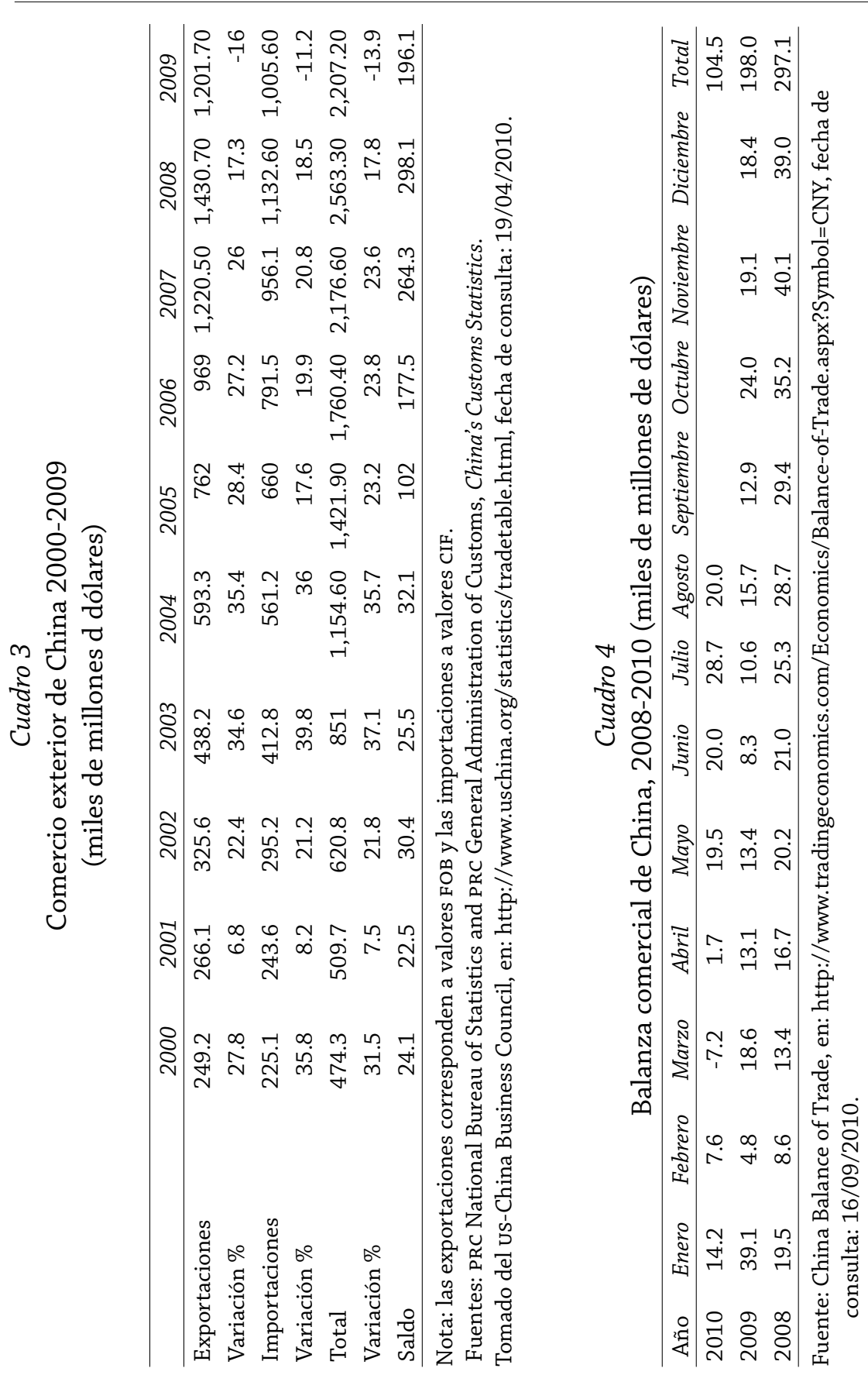


2008. China capta, a nivel mundial, $9.9 \%$ del total de las inversiones. Como resultado de la crisis económica mundial la IED en China disminuyó al igual que en el resto de los países. Como se puede apreciar en el cuadro 5, de 2007 a 2008 la inversión extranjera directa subió de 115.9 a 169.4 mil millones de dólares.

Sin embargo, en 2009 la IED de China bajó a 90 mil millones de dólares y se espera que para 2010 se presente un ligero incremento para alcanzar los 100 mil millones de dólares. De hecho, en los primeros siete meses de 2010 la captación ya había alcanzado los 58.35 mil millones, equivalentes a $20.7 \%$ más que en el mismo periodo de 2009. China permanece como el segundo captador de IED, sólo después de Estados Unidos. En 2009 este país captó 130 mil millones, mientras que China sólo 90 mil millones. ${ }^{12}$

La disminución de la IED en China en 22.34\% de 2007 a 2009 correspondió con la retracción económica global, producto de la crisis; no obstante, fue similar a la lograda en 2006. Como se puede ver en el siguiente cuadro, la IED mundial bajó en $46.9 \%$ de 2007 a 2009; en la economías en desarrollo la disminución fue de sólo $15.3 \%$, mientras que en las economías desarrolladas la caída fue de $60.8 \%$.

Para evitar una caída más pronunciada en la IED, China ha tomado varias medidas, tales como abrir sectores específicos a la inversión extranjera directa, mismos que previamente se habían mantenido cerrados. Dichas medidas incluyen la implementación de nuevas leyes y regulaciones, así como reformas administrativas. Actualmente, la mitad de las exportaciones chinas son producidas por la inversión extranjera directa de carácter manufacturero. ${ }^{13}$

12. Aunque las cifras difieren ligeramente de una fuente a otra, para revisar los datos más actualizados véase: Foxbusiness; http://www.foxbusiness.com/markets/2010/08/16/chinajan-july-fdi-rises-pct-bln/print, fecha de consulta: 17/09/2010; Xinhua, que reporta datos del Ministerio de Comercio de China: http://english.caijing.com.cn/2010-09-06/110514415. html, fecha de consulta: 17/09/2010; Globaltimes: http://business.globaltimes.cn/ world/2010-07/555164.html, fecha de consulta: 17/09/2010.

13. Para más detalles sobre este tema véase: "Foreign Direct Investment in China - Past, Present and Policies", en: http://www.suite101.com/content/foreign-direct-investment-in-china-past-present-and-policies-a238960\#ixzz0zWdFC4VD y en: http://www.suite101.com/content/foreign-direct-investment-in-china--past-present-and-policies-a238960, fecha de consulta: 14/09/2010. 


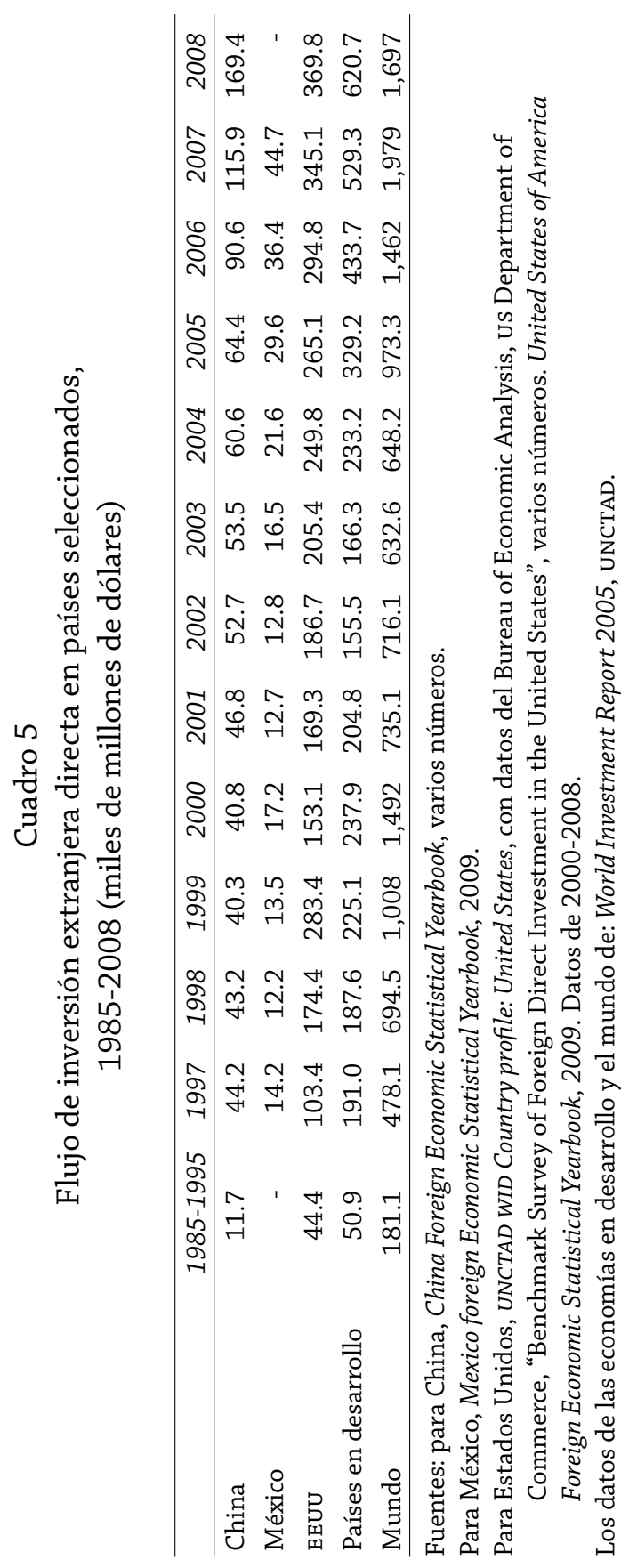

62 México y la Cuenca del Pacífico. Vol. 13, núm. 39/septiembre-diciembre de 2010 


\section{Cuadro 6}

Flujo mundial de inversión extranjera directa, 2007-2009 (miles de millones de dólares)

\begin{tabular}{lrrr}
\hline Año & \multicolumn{1}{c}{2007} & \multicolumn{1}{c}{2008} & \multicolumn{1}{c}{2009} \\
\hline Mundo & 2099973 & 1770873 & 1114189 \\
Economías en desarrollo & 564930 & 630013 & 478349 \\
Economías en transición & 90968 & 122588 & 69948 \\
Economías desarrolladas & 1444075 & 1018273 & 565892 \\
\hline
\end{tabular}

Fuente UNCTAD, Mayor FDI Indicator (WIR, 2010), en: http://stats.unctad.org/FDI/

TableViewer/tableView.aspx?ReportId=4031, fecha de consulta: 17/09/2010.

\section{La política anti-crisis de China}

Ante la crisis financiera global, el gobierno de China actuó rápidamente para mitigar la caída en el crecimiento del PIB a través de un paquete de estímulos y de expansión monetaria. Así, en noviembre de 2008 el gobierno introdujo un paquete de estímulos de cuatro billones de yuanes (14\% del PIB de 2008) para aplicarse en 2009 y 2010. Sólo en el rubro de infraestructura el gobierno chino invirtió 586,000 millones de dólares (NY Times, 2010a).

Una cuarta parte de estos recursos los está otorgando el gobierno central en forma de concesiones (grants) directas a tasas de interés preferenciales (subsidiadas). Los gobiernos locales, por su parte, propusieron su propio paquete de estímulos de 18 billones de yuanes.

La expansión monetaria desde 2009 ha sido también importante para la recuperación de China. El Banco Popular de China ha adoptado una política monetaria muy expansionista para apoyar la política fiscal. En la primera mitad de 2009, la banca de crédito incrementó en 7.3 billones de RMB, el ya sobrepasado objetivo anual (Yu, 2010).

Por su parte el Banco Mundial señala que la fuerte demanda interna fue necesaria para garantizar la recuperación de la economía y por lo mismo requiere probar su grado de sustentabilidad en el largo plazo (Global Crisis News, 2009).

Existe consenso en que la dirigencia china ha mostrado una gran capacidad de reacción frente a la crisis económica mundial. Con la aplicación de una política netamente keynesiana puso en marcha una gran cantidad recursos financieros para apoyar la obra pública. Ante la reducción de las exportacio- 
nes (que hasta hoy ha sido uno de los sustentos de su modelo de desarrollo económico), la reacción ha sido el impulso de la demanda interna, sustentada en el gasto fiscal.

Por otra parte, desde octubre de 2009, indicadores como los precios internos, la producción industrial y las ventas al menudeo mostraban indicios claros de recuperación económica. La inflación que se había incrementado en 2007 debido a la fiebre de inversión y la fuerte demanda externa, para 2009 dejó de ser problema ${ }^{14}$ y la deflación había cedido. Las exportaciones disminuyeron pero en menor medida de lo esperado (IBTIMES, 2009).

Respecto de la producción industrial, cifras oficiales reportaban para los primeros 10 primeros meses de 2009, un incremento de 9.4\% (Xinhua, 2009). Sólo en octubre de 2009, la producción industrial, aumentó $16.1 \%$ en comparación con el mismo mes de 2008; la cifra más alta del año, y por encima de $15.9 \%$ estimada para septiembre. En el mismo mes de octubre la generación de energía subió $17.1 \%$ sobre el mismo mes de 2008, por quinto mes consecutivo (IBTIMES, 2009). Ya en agosto de 2009, The Economist (15 de agosto de 2009) mencionaba que la recuperación del sector industrial de China quedaba de manifiesto en la tasa de crecimiento de $11 \%$ en los doce meses que van de agosto de 2008 a julio de 2009.

En 2009 la producción de acero alcanzó la cifra de 567 millones de toneladas, superior en $13.5 \%$ a la del año anterior. De enero a marzo de 2010 la producción alcanzó las 158 millones de toneladas, cifra que representa un incremento de $24.5 \%$ en relación con el trimestre anterior, lo cual equivale a $46 \%$ de la producción mundial de acero (World Steel Review, 2010).

Cabe recordar que la industria del acero ha sido una de los subsectores "insignia" de los indicadores macroeconómicos de la sobrecapacidad productiva de China. Al respecto, desde 2004 intentó aplicar medidas drásticas sobre los nuevos proyectos de inversión en éste y en otros sectores. En 2004, la producción de acero de China había alcanzado las 400 millones de toneladas, aproximadamente. El gobierno actuó drásticamente sobre la construcción de nuevas siderúrgicas, pero el aumento en la demanda de acero, atribuible al desarrollo de los bienes raíces y la fuerte demanda de exportaciones, propi-

14. Los precios al consumidor, de enero a septiembre, en las 70 ciudades más grandes aumentaron $3.9 \%$, superior en $1.1 \%$ a $2.8 \%$ anual a septiembre de 2008 ("China's industrial output up $16.1 \%$ in Oct.": http://xinhuanet.com). 
ció la construcción de nuevas siderúrgicas. La producción de acero de China aumentó a más de 600 millones de toneladas en 2007 (Yu, 2010).

En 2009 la venta de automóviles en China se elevaron en $46 \%$ respecto del año anterior, alcanzando así una cifra sin precedentes de 13.6 millones de vehículos y convirtiéndose en el mayor mercado del mundo, por encima de los Estados Unidos. Sin embargo, los analistas consideran que los productores de autos chinos difícilmente podrían repetir esta cifra de ventas. ${ }^{15}$

Lo anterior ha sido producto de la política gubernamental de incentivos a la compra de bienes duraderos (Reuters, 2009). En China las facilidades de crédito han sido más importantes que los estímulos fiscales. Esto queda de manifiesto en el hecho de que en los primeros siete meses de 2009 los préstamos bancarios fueron tres veces superiores a los del mismo periodo del año anterior.

El Banco Popular de China informó que en octubre el sistema bancario extendió préstamos por 253,000 millones de yuanes ( $\$ 37,000$ millones de dólares), equivalentes a la mitad de los 516,7000 millones de yuanes (75,600 millones de dólares) otorgados en septiembre. De este modo el total de los préstamos otorgados en este año han totalizado 8.92 billones de yuanes, la mayoría de los cuales se realizaron en la primera mitad del año anterior (IBTIMES, 2009).

La información manejada a los largo de este documento nos permite afirmar que la economía de China ha salido fortificada de la crisis; no sólo en términos económicos sino político-estratégicos, dado que ha incrementado su influencia en la agenda internacional que se maneja en los diversos foros, como son los multilaterales, regionales y bilaterales. Específicamente, en términos económicos, la decisión del gobierno de inyectarle a la economía siete billones de yuanes de préstamos bancarios, el año pasado, adicionales a los cuatro billones de yuanes de estímulos en efectivo, permitió a los exportadores chinos invertir en nuevas plantas; muchas de ellas en las zonas interiores del país donde los costos son menores. Los estímulos en efectivo también permitieron incrementar

15. Hay que tomar en cuenta también que 2009 coincidió con la cifra más bajas de ventas de automóviles en EEUU desde 1982. Los 10.4 millones de autos vendidos en 2009 en este país representan una disminución de $21 \%$ en relación con 2008 . Además, las cifras no incluyen la venta de vehículos comerciales pesados, que fue de 500,000 unidades. Véase: The Wall Street Journal, “China Passes U.S. as World's Top Car-Market”, enero 12, 2010: http://online.wsj. com/article/SB1000142405274870365210457465183 3126548364.html, fecha de consulta: 25/09/2010. Chinaview, 2009. 
sustancialmente las inversiones en caminos, vías férreas e infraestructura, que incidirán en la disminución de los costos y en una mejor integración económica nacional. De las crisis surgen nuevos retos; de ellos se puede salir fortificado si se toman las medidas pertinentes. Este es el caso de China.

\section{Conclusiones}

Es posible que lo peor de la crisis de los mercados financieros y de la economía mundial en su conjunto ya haya pasado. Sin embargo, existen suficientes riesgos para considerar que los tomadores de decisiones y los mercados no pueden relajarse. La política pública requiere de la aplicación de algunas medidas ya diseñadas para el control de los sectores financiero y económico en general. La superación de la crisis y la recuperación de una senda de crecimiento requieren de programas sólidos (Baily y Douglas).

La crisis económica mundial tuvo elementos comunes con otras del pasado; la especulación desmedida por falta de controles gubernamentales se ha definido como una de las causas fundamentales. Una característica particular de esta crisis fue que afectó a una gran parte del mundo de manera simultánea. Por tal razón, fue necesario que las economías que originaron la crisis aplicaran medidas correctivas para hacerle frente. Los resultados positivos, obtenidos relativamente rápido, están creando las condiciones para una recuperación del sector financiero mundial y posteriormente de la economía real.

Teniendo en cuenta algunos indicadores básicos de la economía de China se puede afirmar que no obstante la recesión mundial acontecida en 2008 y 2009, China logró mantener niveles de crecimiento envidiables para la mayoría de las economías del mundo.

La recuperación económica de 2009 en buena medida se debe a las grandes inversiones gubernamentales, los apoyos al consumo y los estímulos a la inversión productiva. Las perspectivas de una reactivación en las exportaciones para 2010 constituyen un complemento a las medidas keynesianas vigentes. Sin embargo, ello no implica que haya superado los rezagos socioeconómicos de una buena parte de la población, ni los problemas estructurales de una economía depredadora, que afecta sobre todo al medio ambiente.

En el contexto de una reactivación económica de la economía mundial, China ha salido fortificada del proceso. Por una parte su economía ha sido una de las menos afectadas; por la otra, su relación productiva con las economías 
del este de Asia le ha conferido un estatus de liderazgo en el manejo de los asuntos económicos y estratégico-políticos de la región.

\section{Bibliografía}

Banco Mundial, Perspectivas para la Economía Mundial. Crisis, finanzas y crecimiento, Washington, DC, 2010.

Drysdale, Peter, "Politics and Chinese integration into the global economy", East Asia Forum, 4 de abril de 2010.

IMF (2009), World Economic Outlook. Crisis and Recovery, Washington, DC, abril, 2009.

Baily, Martin Neil y Douglas J., Elliot, "The us Financial and Economic Crisis: Where Does it Stand and Where Do We Go From Here?", Business and Public Policy at Brookings, Washington, DC, junio, 2009.

Blair, C. Dennis, "Annual Threat Assessment of the Us Intelligence Community for the Senate Select Committee on Intelligence", Office of the Director of National Intelligence, EEUU, 2010.

Gilpin, Robert (1996), "The Nature of Political Economy”, en Art, C. Robert y Jervins, Robert, International Politics. Enduring Concepts and Contemporary Issues, Harper Collins College Publishers, Fourth Edition, Nueva York, pp. 269-285.

Krugman, Paul (2008), "La crisis paso a paso", en Paul Krugman et al., "La crisis económica mundial”, La Oveja Negra, Bogotá, Colombia, pp. 31-51.

Lardy, R. Nicholas, Integrating China into the Global Economy, Brookings Institution Press, Washington, DC, 2002.

Salama, Pierre, "Una crisis financiera estructural", Iconos. Revista de Ciencias Sociales, núm. 36, Quito, enero de 2010, pp. 19-28.

Soros, George (2008a), El nuevo paradigma de los mercados financieros. Para entender la crisis económica actual, Taurus, Madrid, España.

Stiglitz, Joseph, "La caída de Wall Street es para el fundamentalismo del mercado lo que la caída del muro de Berlín lo fue para el comunismo", en Paul Krugman et al., La crisis económica mundial, La Oveja Negra, Bogotá, Colombia, 2008, pp. 61-62.

Sutter, Robert (2009), "The Obama Administration and us Policy in Asia", Contemporary Southeast Asia, vol. 31, núm. 2, pp. 189-216.

The Economist, 15 de agosto, 2009, p. 69.

The World Bank, Global Economic Prospects (2010). 
United Nations (2010), World Economic Situation and Prospects 2010, Nueva York.

Zheng, Yongniean (2004), Globalization and State Transformation in China, Cambridge University Press, p. xv.

Fuentes electrónicas

Chinability 2010, en: http://www.chinability.com/GDP.htm, fecha de consulta: 07/09/2010.

China Balance of Trade 2010, en: http://www.tradingeconomics.com/ Economics/Balance-of-Trade.aspx?Symbol=CNY, fecha de consulta: 16/09/2010.

Chinaview 2009, “China's economic growth to top 9\% next year”, en Chinaview, en: mofcon.com.gov.cn, fecha de consulta: 20/11/2009.

CIA, The World Fact Book, China, en: https://www.cia.gov/library/publications/ the-world-factbook/geos/ch.html, fecha de consulta: 10/05/10.

FAO, "Undernourishment around the world", París, 2009, p. 9, en: ftp://ftp.fao. org/docrep/ fao/012/i0876e/i 0876e02.pdf, fecha de consulta:12/05/10.

Global Crisis News, en http://www.globalcrisisnews.com/Asia/, fecha de consulta: 15/11/2009.

IMF (2010), “World Economic Outlook Update. Restoring Confidence without Harming Recovery”, Washington, DC, julio, 2010, en: http://www.imf.org/ external/pubs/ft/weo/ 2010/update/02/index.htm, fecha de consulta: 6/09/2010.

International Business Times (IBTIMES), 2009, en: http: //www.ibtimes.com. au/, fecha de consulta: 15/11/2009.

Los Angeles Times (LA Times), 2010, Bloomberg News, septiembre 20, 2010, en: http://www.latimes.com/business/la-fi-recession-20100920,0,4014811. story, fecha de consulta: 23/09/2010.

Lan, Sue y Sara Cook, "China and the Global Financial Crisis: Implications for Low-income Countries", Workshop co-organized by Brookings-Tsinghua Centre, Beijing, funded by the Uk Department for International Development (DFID), marzo, 2009, en: http://www.brookings.edu/ /media/Files/ events/2009/0320_china_financial_crisis/0320_china_financial_crisis_remarks.pdf, fecha de consulta: 15/05/2010.

NationMaster.com. Economy, en: http://www.nationmaster.com/graph/ eco_gdp-economy-gdp\&date=2006, fecha de consulta: 17/05/2010. 
New York Times (NY Times), 2010a, en: http://www.nytimes.com/2010/08/16/ business/global/ 16yuan.html, fecha de consulta: 08/09/2010.

New York Times (NY Times), 2010b, "Is This What a Recovery Feels Like?", septiembre 20, 2010, en: http://www.nytimes.com/roomfordebate/2010/09/20/is-this-what-a-recovery-feels-like, fecha de consulta: 23/09/2010.

Reuters, 2009, en; http://www.reuters.es, fecha de consulta: 16/11/2009.

The Economist, 2010, "Second in line", 16 de agosto de 2010, en: http:// www.economist.com/blogs/freeexchange/2010/08/china_0, fecha de consulta: 01/09/2010.

White, William, "Modern Macroeconomics Is on the Wrong Track", Finance and Development, diciembre de 2009, en: http://www.imf.org/external/ pubs/ft/fandd/2009/12/pdf/ white.pdf, fecha de consulta: 22/05/2010. World Economic Outlook Database, 2010, International Monetary Fund, en: http://www.imf.org/external/pubs/ft/weo/2010/01/weodata/weorept. aspx? sy $=1990 \&$ ey $=1999 \& \mathrm{scsm}=1 \& \mathrm{ssd}=1 \&$ sort $=$ country $\& \mathrm{ds}=. \& \mathrm{br}=1$ $\& c=924 \& s=P P P P C \& g r p=0 \& a=\& p r 1 . x=59 \& p r 1 . y=5$, fecha de consulta: $31 / 05 / 2010$.

World Steel Review (2010), "World Steel Production Report. ISSB Monthly World Steel Production Review", World Steel Review, mayo, 2010, en: http://www.steelonthenet.com/production.html, fecha de consulta: 20/05/2010; y en: http://minerals-and-metals.blogspot.com/2010/01/ chinas-steel-production-for-2009-567mn.html).

The World Bank, 2010, Global Economic Prospects (2010), en: http://web. worldbank.org/ WBSITE/ EXTERNAL/EXTDEC/EXTDECPROSPECTS/ EXTGBLPROSPECTSAPRIL/ 0,,menuPK:659178 pagePK:64218926 piPK:64218953 theSitePK:659149,00.html), junio 10, 2010, fecha de consulta: 31/08/2010.

The Wall Street Journal Asia, 2010, “China Passes us as World's Top Car-Market", enero 12, 2010, en: http://online.wsj.com/article/ SB100014240527487036521045746 51833126548364.html, fecha de consulta: 25/09/2010.

Xinhua, 2009, “China's industrial output up 16.1\% in Oct. 2009”, Xinhua, en: http://xinhuanet.com.

Xinhua, 2010, en: http://english.peopledaily.com. cn/90001/90778/90861/6965352.html, fecha de consulta: 14/09/2010. 
Yu, Yongding, "China's Policy Responses to the Global Financial Crisis", China, Economic Policy, Financial crisis, Comments, East Asian Forum, 24, January 2010, en: http://www.eastasiaforum.org, fecha de consulta: 08/05/10.

Yu, Yongding, Foreign Direct Investment in China - Past, Present and Policies, en: http://www.suite101.com/content/foreign-direct-investment-in-china-past-present-and-policies-a238960\#ixzz0zWdFC4VD; y en: http://www. suite101.com/content/foreign-direct-investment-in-china--past-presentand-policies-a238960, fecha de consulta: 14/09/2010. 